\title{
Probing the ultrafast electron and lattice dynamics of gold using femtosecond mid-infrared pulses
}

\author{
Harmen J. Sielcken $\oplus^{1,2}$ and Huib J. Bakker ${ }^{1, *}$ \\ ${ }^{1}$ AMOLF, Science Park 104, 1098 XG Amsterdam, The Netherlands \\ ${ }^{2}$ ARCNL, Science Park 110, 1098 XG Amsterdam, The Netherlands
}

(Received 29 August 2018; revised 24 August 2020; accepted 10 September 2020; published 6 October 2020)

\begin{abstract}
We study the ultrafast electron and lattice dynamics of thin gold films with mid-infrared transmission and reflection pump-probe spectroscopy. We determine the time dependence of the dielectric function, which yields the electron-phonon coupling constant $G$ that determines the energy exchange rate between the electrons and the lattice. From the experimentally obtained dielectric function, we determine the dependence of the electronelectron and the electron-lattice scattering rates on the electron and the lattice temperature.
\end{abstract}

DOI: 10.1103/PhysRevB.102.134301

\section{INTRODUCTION}

The study of ultrafast light-metal interactions has been a topic of interest for a long time, from both theoretical and practical points of view. From a theoretical point of view this topic is highly interesting in view of the fundamental physical processes that take place in metals at ultrafast time scales. Examples of these processes are electron thermalization [1-4], thermoelectric heat transport [5-7], and propagation and excitation of coherent phonons [8,9].

One of the key aspects of ultrafast light-metal interaction is the creation of a transient nonequilibrium state between the electrons and the lattice. Kaganov et al. [10] showed that when a conductor is heated by a rapidly oscillating electric field, such as a femtosecond laser pulse, conduction electrons are excited by this field, while the lattice initially remains cool. The excited electrons thermalize $[4,11]$ and cool by energy transfer to the lattice $[12,13]$. These thermalization and cooling dynamics change the optical $[13,14]$ and thermal properties [15] of the metal on a femtosecond timescale.

The understanding of the evolution of the nonequilibrium state is very important as it makes it possible to exploit these transient properties in technological applications. For example, the transient nonequilibrium state plays an important role in laser cutting and in the processing of materials [15-17]. When an intense laser pulse hits a material surface, the leading edge of the pulse can alter the optical properties of the material with the result that the trailing part undergoes a very different interaction with the material. This may lead to an increase in the fraction of absorbed energy of the laser pulse by the material [18].

In this paper we investigate the properties of gold following ultrafast optical excitation using transient reflection (TR) and transient transmission (TT) measurements in the mid-infrared. The use of mid-infrared probing pulses allows for a selective measurement of the electron dynamics within the conduction band that allows for an accurate determination of the electronelectron and electron-phonon scattering rates.

\footnotetext{
*h.bakker@amolf.nl
}

\section{METHODS}

We measure the electron and lattice dynamics of a thin gold film on a $\mathrm{CaF}_{2}$ substrate. The sample is made by thermal deposition at a pressure of $1 \times 10^{-6} \mathrm{mbar}$. The resulting layer is inspected with an atomic force microscope and shows a thickness of $17 \pm 1 \mathrm{~nm}$. The AFM images show that the film is uniform and continuous with an rms roughness of $\sim 1 \mathrm{~nm}$ (averaged over a strip of $5 \times 0.1 \mu \mathrm{m}^{2}$ ). Some small surface features are visible, which are reminiscent of the percolation structure that is present below the percolation threshold (i.e., a nominal evaporated thickness of $10 \mathrm{~nm}$ ) [19].

The linear optical properties of the film are determined using ellipsometry in the wavelength range from 0.7 to $1.4 \mu \mathrm{m}$. The optical properties of the film are in accordance with those of thicker films, as reported in Sec. III B 1. We also perform Fourier transform infrared experiments in transmission to obtain the optical response of the film at mid-infrared wavelengths up to $10 \mu \mathrm{m}$. The measured transmission spectrum does not show any shape-dependent resonances or other anomalies. We therefore conclude that the film can be characterized as a uniform thin film with an unknown with an unknown orientation of the crystalites.

In the femtosecond experiments we excite the sample with a strong femtosecond laser pulse (pump), and we monitor the TT and TR of the sample with a weak femtosecond probing pulse as a function of the time delay between the pump and the probe. We define the TR/TT signal as follows:

$$
\begin{aligned}
& \Delta T(t)=\frac{T(t)}{T_{0}}-1, \\
& \Delta R(t)=\frac{R(t)}{R_{0}}-1,
\end{aligned}
$$

where $T(t)$ and $R(t)$ are the transmittance and reflectance as a function of the delay $t$ between the pump and probe and $T_{0}$ and $R_{0}$ are the unperturbed transmittance and reflectance.

To minimize the effect of the scattering of the pump light from the sample on the TT and TR signals, we perform a twocolor pump-probe experiment in which we tune the photon energy of the pump and probe independently. The pump and probe are generated by two optical parametric amplifiers. The 

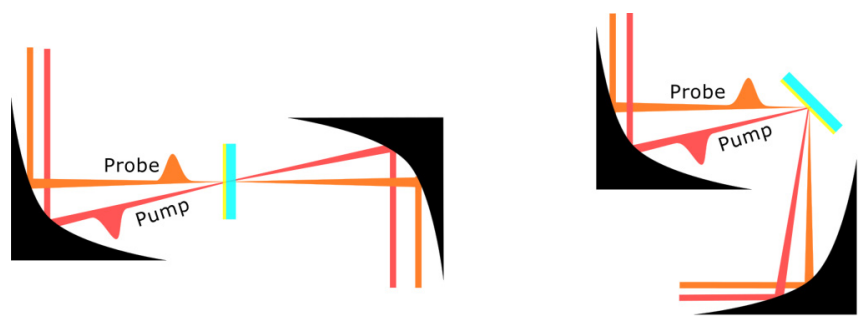

FIG. 1. The experimental geometries used in the (left) TT and (right) TR experiments. The frequencies of the pump and probe are $0.42 \mathrm{eV}$ (0.015 eV FWHM) and $0.34 \mathrm{eV}$ (0.03 eV FWHM), respectively.

pump is tuned at a photon energy of $0.42 \mathrm{eV}$ with a bandwidth of $0.015 \mathrm{eV} \mathrm{FWHM,} \mathrm{corresponding} \mathrm{to} \mathrm{a} \mathrm{central} \mathrm{wavelength}$ of $2.95 \mu \mathrm{m}$ with a width of about $0.1 \mu \mathrm{m}$ FWHM. The probe has a photon energy of $0.34 \mathrm{eV}$ and a bandwidth of $0.03 \mathrm{eV}$ FWHM, corresponding to a central wavelength of $3.65 \mu \mathrm{m}$ with a width of about $0.3 \mu \mathrm{m}$ FWHM. A cross-correlation measurement between the pump and the probe in a 2-mmthick Ge window yields a pulse width of about $120 \mathrm{fs}$. The pump and probe pulses are focused on the sample by a gold parabolic mirror with a focal distance of $10 \mathrm{~cm}$ to a spot with a diameter of $\sim 240 \mu \mathrm{m}$ FWHM and $\sim 120 \mu \mathrm{m}$ FWHM, respectively. After the sample, the probe pulse is collimated, dispersed by a spectrograph, and detected using a 32-pixel mercury cadmium telluride (MCT) array. A more detailed description of the IR generation and data acquisition setup is given elsewhere [20].

The time-resolved experiments are performed at a repetition rate of $1 \mathrm{kHz}$, and we chop every other excitation pulse, meaning that the repetition rate of the pump is $500 \mathrm{~Hz}$. No transient signal is observed at negative time delays, which implies that the thermal equilibration is complete within $2 \mathrm{~ms}$.

The TT and TR experiments are performed separately. The TT measurements are probed at normal incidence, whereas the TR measurements are probed at a $45^{\circ}$ angle of incidence with a $p$-polarized probe. The experimental geometries are shown in Fig. 1.

The time-dependent TT and TR signal can be converted to a transient change in the complex dielectric function of gold $\tilde{\varepsilon}$ using the relations between the transmittance $T$, the reflectance $R$, and $\tilde{\varepsilon}$ :

$$
\begin{gathered}
T=\left|\frac{t_{a, g} t_{g, c} e^{i \tilde{n}_{g} \frac{\omega}{c} d}}{1-r_{g, c} r_{g, a} e^{i 2 \tilde{n}_{g} \frac{\omega}{c} d}}\right|^{2}, \\
R=\left|r_{a, g}+\frac{t_{a, g} r_{g, c} t_{g, a} e^{i 2 \tilde{n}_{g} \frac{\omega}{c} d \cos \theta_{t}}}{1-r_{g, c} r_{g, a} e^{i 2 \tilde{n}_{g} \frac{\omega}{c} d \cos \theta_{t}}}\right|^{2}, \\
r_{1,2}=\frac{\tilde{n}_{2} \cos \theta_{i}-\tilde{n}_{1} \cos \theta_{t}}{\tilde{n}_{1} \cos \theta_{t}+\tilde{n}_{2} \cos \theta_{i}}, \\
t_{1,2}=\frac{2 \tilde{n}_{1} \cos \theta_{i}}{\tilde{n}_{1} \cos \theta_{t}+\tilde{n}_{2} \cos \theta_{i}},
\end{gathered}
$$

with $\tilde{n}_{a}, \tilde{n}_{g}$, and $\tilde{n}_{c}$ being the complex refractive indices of air, gold, and $\mathrm{CaF}_{2}$, respectively; $\theta_{i}$ and $\theta_{t}$ being the angles of incidence and transmittance; $d$ being the thickness of the sample; $\omega$ being the frequency of the probe; $c$ being the speed of light; and $t_{1,2}$ and $r_{1,2}$ being the Fresnel transmission and reflection coefficients of the respective interface. In our experiment $\tilde{n}_{a}=1.00, \tilde{n}_{g}=1.17+26.10 i$, and $\tilde{n}_{c}=1.41[21]$, and $\theta_{i}=45^{\circ}$. The complex angle of transmittance $\theta_{t}$ is related to the angle of incidence $\theta_{i}$ via Snell's law.

The complex refractive index $\tilde{n_{g}}=n_{g}+i \kappa_{g}$ is directly related to the complex dielectric function of gold:

$$
\tilde{n}_{g}=n_{g}+i \kappa_{g}=\sqrt{\varepsilon_{g, r}+i \varepsilon_{g, i}}=\sqrt{\tilde{\varepsilon}_{g}},
$$

with $\varepsilon_{g, r}$ and $\varepsilon_{g, i}$ being the real and imaginary parts of the dielectric function of gold, respectively.

\section{RESULTS}

\section{A. Femtosecond transmission and reflection measurements}

Figure 2 shows the transient transmission and reflection change as a function of the delay between the pump and probe pulses for several pump intensities. Time delay zero is defined as the delay point after the rapid change in the TT and TR signals.

At time delay zero, the transmittance shows a strong increase, while the reflectance shows a strong decrease. These abrupt changes in the optical properties are caused by the heating of the conduction electrons of the gold film. The rapid changes are followed by slower dynamics on a picosecond timescale. These slower dynamics reflect the cooling of the electrons and heating of the lattice by energy transfer to the lattice, until thermal equilibrium between the electronic and lattice systems is reached [10].

\section{B. Time-dependent dielectric function and two-temperature model}

The TT and TR signals of Fig. 2 follow from changes in the dielectric function that in turn follow from the evolution of the electron and lattice temperatures that are induced by the excitation of the gold film. Hence, to interpret the data of Fig. 2, we will establish the relation between the dielectric function and the electron and lattice temperatures, and we will model the evolution of these temperatures in the gold film after the excitation.

\section{Intraband and interband dielectric response}

The linear optical properties of metals in the infrared can be well accounted for by the Drude model [22]. This model includes the dielectric response of the conduction electrons (intraband transitions) and the nonresonant response of the bound electrons (interband transitions), which usually have transitions in the visible and $\mathrm{UV}$, and is given by

$$
\begin{gathered}
\tilde{\varepsilon}=\varepsilon_{\infty}-\frac{\omega_{p}^{2}}{\omega(\omega+i \gamma)}, \\
\gamma=\gamma_{e p}+\gamma_{e e},
\end{gathered}
$$

where $\omega_{p}$ is the plasma frequency, $\varepsilon_{\infty}$ is the nonresonant background dielectric response due to bound electrons, and $\gamma_{e p}$ and $\gamma_{e e}$ are the electron-phonon and electron-electron scattering rates.

To obtain the Drude parameters of our gold sample at room temperature $(300 \mathrm{~K})$, we fit the dielectric function of the film $\tilde{\varepsilon}_{g}$ that we determined with ellipsometry to Eq. (8) and obtain 


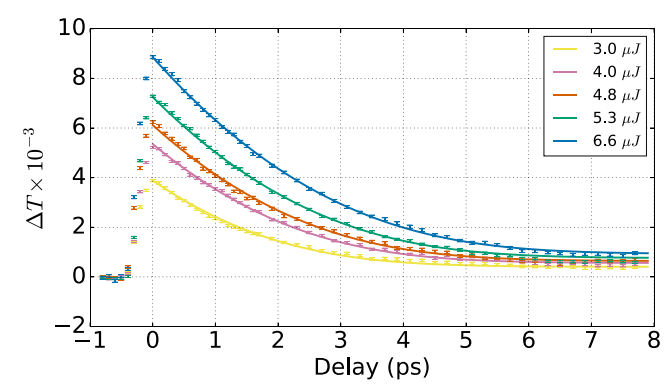

(a)

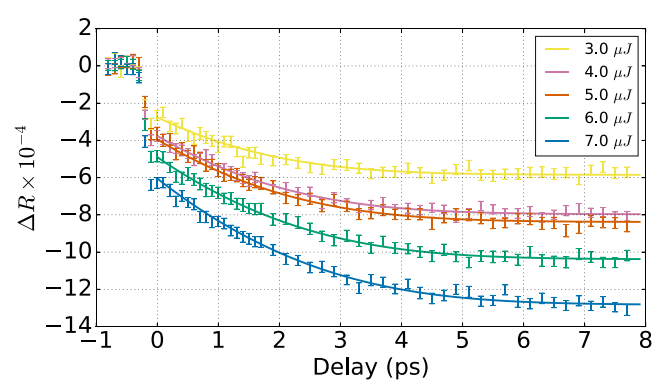

(b)

FIG. 2. Transient (a) transmission and (b) reflection measurements for different pump intensities on a 17-nm-thick gold film when probed at $0.34 \mathrm{eV}$. The solid lines are fits obtained using the procedure in Sec. III B 2 .

$\hbar \omega_{p}=8.81 \mathrm{eV}, \hbar \gamma=0.03 \mathrm{eV}$, and $\varepsilon_{g, \infty}=7.24$. These values agree with previously reported values [23].

It is well known that the nonresonant background dielectric response and the scattering rates depend on both the lattice and electron temperatures. The nonresonant background represents the response of the interband transitions. These are the electronic transitions from the valence band ( $d$ band) to the top of the conduction band ( $s / p$ band), which is defined as the Fermi level [22]. These transitions start absorbing around $2.4 \mathrm{eV}$ [23], above the interband transition threshold.

Due to the Fermionic nature of the electrons, these transitions can occur only if a state in the $s / p$ band is empty [22]. The occupancy of the $s / p$ band is determined by the FermiDirac distribution [24]. When the conduction electrons are heated, electrons below the Fermi level are thermally excited, leaving empty states below the Fermi level while filling states above the Fermi level. This is called Fermi smearing. Due to this smearing, there is an increased absorption below $2.4 \mathrm{eV}$ and a decreased absorption above $2.4 \mathrm{eV}$ [13].

A second effect that causes the occupancies to change is that of Fermi shifting [24]. This is the effect in which the Fermi level decreases with increasing electron temperature. This shifting leads to fewer occupied states below the unperturbed Fermi level (i.e., the Fermi level at $300 \mathrm{~K}$ ), thus leading to a decrease of the interband transition threshold. Using a Kramers-Kronig transformation [22], it can be shown that the Fermi smearing and shifting lead to an electron temperaturedependent nonresonant background [4]

$$
\Delta \varepsilon_{g, \infty}=a\left(T_{e}^{2}-300 \mathrm{~K}^{2}\right),
$$

where $a$ is a constant that has yet to be determined. Schoenlein et al. did transient reflection experiments at optical frequencies [25] to study the temperature dependence of the electronic occupancies in a temperature range similar to that in our experiments. In this study a similar decrease of the electron temperature after ultrafast optical excitation was observed as a result of cooling to the lattice. This study also showed that the interband transitions are primarily affected by Fermi smearing and hardly by Fermi shifting.

The electron-electron scattering rate also changes with the electron temperature because this rate depends on the width of the Fermi-Dirac distribution, as the electrons with an energy within $k_{B} T$ around the Fermi level are the only electrons that can participate efficiently in electron-electron scattering events [24].
Lattice vibrations, or phonons, are the main source of electron-lattice scattering events at room temperature. Above the Debye temperature, the number of scattering events is proportional to the number of phonon excitations. As the number of phonon excitations is proportional to the lattice temperature, the electron-lattice scattering rate is also proportional to the lattice temperature [24].

It can be shown that for electronic temperatures up to $3000 \mathrm{~K}$ and lattice temperatures up to $1200 \mathrm{~K}$ the following proportionalities hold $[26,27]$ :

$$
\Delta \gamma=\Delta \gamma_{e p}+\Delta \gamma_{e e}=b\left(T_{l}-300 \mathrm{~K}\right)+c\left(T_{e}^{2}-300 \mathrm{~K}^{2}\right),
$$

where $b$ and $c$ are constants to be determined.

\section{Two-temperature model}

Using Eqs. (10) and (11), we can relate the transient dielectric function to the dynamics of the electron and lattice temperatures. These dynamics can be accounted for by the so-called two-temperature model (TTM) that describes the energy exchange between the electrons and the lattice [10]. The TTM describes the system of conduction electrons and the lattice as two separate heat baths that are coupled via the electron-phonon coupling:

$$
\begin{aligned}
C_{e} T_{e} \frac{d T_{e}}{d t} & =-G\left(T_{e}-T_{l}\right), \\
C_{l} \frac{d T_{l}}{d t} & =G\left(T_{e}-T_{l}\right),
\end{aligned}
$$

with $C_{e}$ being the electronic heat capacity constant, $C_{l}$ being the lattice heat capacity, $T_{e}$ and $T_{l}$ being the electron and lattice temperatures, respectively, and $G$ being the electron-phonon coupling constant.

This model assumes that at all delay times the lattice and electronic systems can be fully characterized with distinct temperatures. This means that the thermodynamics of the lattice are described by Maxwell-Boltzmann statistics, whereas that of the electronic system is described by Fermi-Dirac statistics. In our description of the TTM, we neglect electron diffusion, as the thickness of the film is on the order of the penetration depth of the pump pulse and we can therefore assume that the system is uniformly heated.

The TTM does not account for the initial nonthermal phase of the electron system that is induced by the femtosecond optical excitation. It has been shown that the electronic system can be nonthermal up to 2 ps [4]. The thermalization time of 
the electronic system is strongly dependent on the used pump fluence and photon energy and is, in our case, always expected to be smaller than 250 fs [4]. This is not within the duration of the pump pulse. However, as the pump frequency $(0.42 \mathrm{eV})$ is small with respect to the Fermi energy of gold $(5.53 \mathrm{eV})$ [24], the electron system will not be far from thermal equilibrium. Therefore, the studied system can be well described at all times with an electron and a lattice temperature.

In order to relate the TT signal to the electron and lattice temperatures, we need an expression for the transmittance as a function of these temperatures. From the Drude model, it follows that $\varepsilon_{g, \infty}$ and $\gamma$ are affected by the temperature dynamics. Assuming that $\Delta \tilde{\varepsilon}$ is small compared to the unperturbed $\tilde{\varepsilon}$, we can expand $T(t)$ around the unperturbed dielectric function in $\varepsilon_{g, \infty}$ and $\gamma$, yielding

$$
\begin{aligned}
\Delta T= & \frac{\partial T}{\partial \varepsilon_{g, \infty}} \Delta \varepsilon_{g, \infty}+\frac{\partial T}{\partial \gamma} \Delta \gamma, \\
\Delta T= & {\left[a \frac{\partial T}{\partial \varepsilon_{g, \infty}}+c \frac{\partial T}{\partial \gamma}\right]\left(T_{e}^{2}-300 \mathrm{~K}^{2}\right) } \\
& +\left[b \frac{\partial T}{\partial \gamma}\right]\left(T_{l}-300 \mathrm{~K}\right), \\
\Delta T= & \alpha\left(T_{e}^{2}-300 \mathrm{~K}^{2}\right)+\beta\left(T_{l}-300 \mathrm{~K}\right) .
\end{aligned}
$$

The values of $\alpha$ and $\beta$ can be determined from the TT experiments. The initial TT signal ( 0 ps) is due to only the heating of the electron temperature, assuming that a negligible amount of energy is exchanged between the electrons and lattice during the excitation process. This is a good assumption as the pulse length is $\sim 80$ times shorter than the time it takes to equilibrate the electrons with respect to the lattice. After relaxation ( $8 \mathrm{ps}$ ), the TT signal is mainly due to lattice heating because almost all of the pump energy has been transferred to the lattice. The change in the internal energy of a free-electron gas is $\Delta U_{e}=\frac{1}{2} C_{e}\left(T_{e}^{2}-300 \mathrm{~K}^{2}\right)$, and the change in internal energy of the lattice is $\Delta U_{l}=C_{l}\left(T_{l}-300 \mathrm{~K}\right)$. As the TT signal shows the same dependence on $T_{e}$ and $T_{l}$ as the internal energy, and as the change in the internal energy $\Delta U$ is proportional to the excitation energy, we expect that both the initial and final TT signals scale linearly with the excitation energy.

We obtain $\alpha$ and $\beta$ by varying the pump energy and fitting the initial and final signals as a function of the intensity to a linear function. The results and fits are shown in Fig. 3. The initial and final signals are a linear function of the excitation energy, as anticipated.

To obtain the electron-phonon coupling constant $G$, we first calculate the electron temperature after excitation using the measured values of the pulse energy, spot size, and absorbed fraction of the excitation energy at the wavelength of the pump, also called the absorptance $A=1-T-R=0.007$. Subsequently, we determine $G$ by fitting the TT measurements using the TTM temperature dynamics and Eq. (14). We use $C_{l}=2.43 \mathrm{MJ} \mathrm{m}^{-3} \mathrm{~K}^{-1}$ and $C_{e}=62.9 \mathrm{~J} \mathrm{~m}^{-3} \mathrm{~K}^{-2}$ [13]. The fits are shown in Fig. 2(a) and are in excellent agreement with the TT measurements. We obtain $G=1.97 \pm 0.15 \times$ $10^{16} \mathrm{~W} \mathrm{~m}^{-3} \mathrm{~K}^{-1}$. In Fig. 4 the transient electron and lattice temperatures that result from the fit of the TTM to the TT measurements are shown.

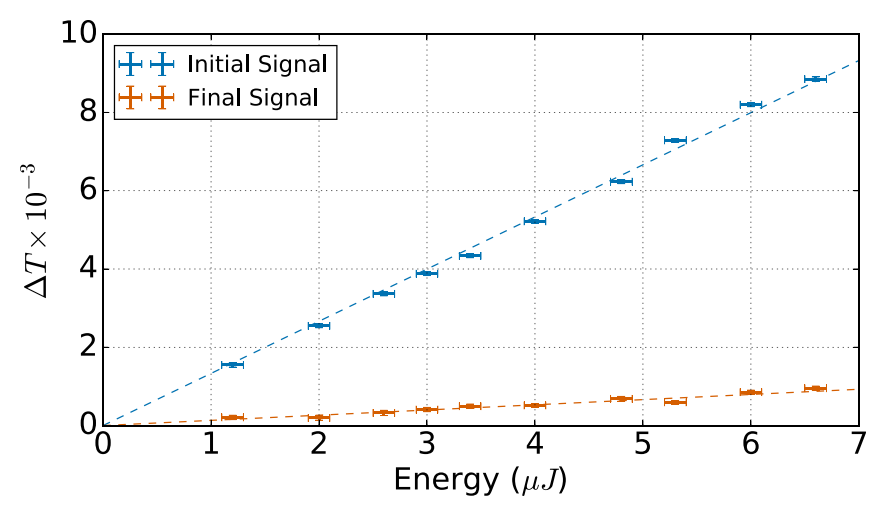

FIG. 3. The initial and final TT signals as a function of excitation energy. The dashed lines are linear fits.

The transient reflection data are measured with a different experimental geometry than the transient transmission data, which implies that the initial electron temperature may not be the same as in the transmission experiment with the same excitation pulse energy. To be able to determine the dielectric function from the TT and TR data, we need pairs of transmission and reflection measurements under the same excitation conditions, i.e., the same initial electron temperature. Hence, we measure a large series of TR data with different excitation energies. Subsequently, we model these TR data with the TTM model using the value of the electron-phonon coupling constant $G$ determined from the TT data and using the initial electron temperature as a fit parameter. With this approach we can identify pairs of TT and TR measurements that have the same initial electron temperature. In Fig. 2(b) we show the set of TR measurements that most closely corresponds to the TT measurements of Fig. 2(a).

\section{Determination of the transient dielectric function}

We determine the transient dielectric function of the gold film from pairs of TR and TT signals that have the same initial electron temperatures. The TT and TR signals are converted into $\Delta \tilde{\varepsilon}$ using Eqs. (3), (4), and (7). The results of this conversion are shown as the data points in Fig. 5.

The dielectric function of Eq. (8) depends on the electron and lattice temperatures through the electron-electron scattering rate $\gamma_{e e}$, the electron-phonon scattering rate $\gamma_{e p}$, and the nonresonant interband dielectric response $\varepsilon_{g, \infty}$. Using the time dependence of the electron and lattice temperatures as given by Fig. 4, we can now fit the parameters $a, b$, and $c$ that describe the dependence of $\gamma_{e e}, \gamma_{e p}$, and $\varepsilon_{g, \infty}$ on the electron and lattice temperatures. We perform this fitting procedure for each initial electron temperature independently and obtain the following parameters:

$$
\begin{aligned}
& a=\varepsilon_{g, \infty}\left(1.93 \times 10^{-7} \pm 3 \% \mathrm{~K}^{-2}\right), \\
& b=\gamma_{0}\left(3.18 \times 10^{-3} \pm 2 \% \mathrm{~K}^{-1}\right), \\
& c=\gamma_{0}\left(1.62 \times 10^{-8} \pm 6 \% \mathrm{~K}^{-2}\right),
\end{aligned}
$$

where $\gamma_{0}$ is the electron-scattering rate at $300 \mathrm{~K}\left(\gamma_{0}=\right.$ $0.03 \mathrm{eV})$. The resulting real and imaginary dielectric functions are shown in Fig. 5 as the solid lines and are in very good 


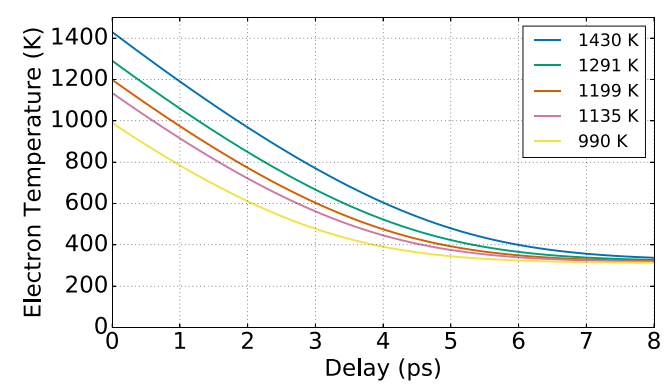

(a)

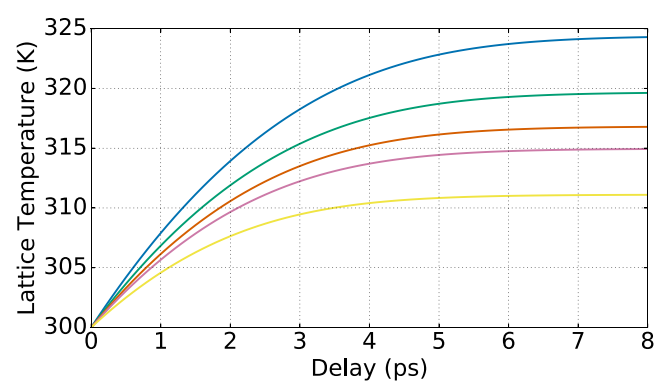

(b)

FIG. 4. The evolution of (a) the electron and (b) the lattice temperature for different excitation energies, as modeled by the two-temperature model with a fitted electron-phonon coupling constant $G=1.97 \pm 0.15 \times 10^{16} \mathrm{~W} \mathrm{~m}^{-3} \mathrm{~K}^{-1}$.

agreement with the data points obtained from the TT and TR measurements.

It is clear from Fig. 5 that $\Delta \varepsilon_{g, i}$ and $\Delta \varepsilon_{g, r}$ display dynamics on the same timescale as the TT and TR data in Fig. 2, but in a different direction. $\Delta \varepsilon_{g, r}$ shows a decay returning almost to zero after $\sim 8 \mathrm{ps}$, whereas $\Delta \varepsilon_{g, i}$ show a further ingrowth, reaching a maximum final value at $\sim 8 \mathrm{ps}$. We also note that the dynamics of $\Delta \varepsilon_{g, r}$ are very similar to those of the TT signal, whereas the dynamics of $\Delta \varepsilon_{g, i}$ resemble those of the TR signal. This may seem surprising, as a change in $\varepsilon_{g, i}$ is usually associated with a change in extinction $k$ and thus the transmittance. This is, however, not the case for metals when probing in the mid-infrared and can be understood as follows. By taking the square of Eq. (7) and replacing $n_{g}$ by $n_{g}+\Delta n_{g}$ and $\kappa_{g}$ by $\kappa_{g}+\Delta \kappa_{g}$, where $\Delta n_{g}$ and $\Delta \kappa_{g}$ have the same order of magnitude, we obtain

$$
\begin{aligned}
& \Delta \varepsilon_{g, r} \simeq 2 n_{g} \Delta n_{g}-2 \kappa_{g} \Delta \kappa_{g}, \\
& \Delta \varepsilon_{g, i} \simeq 2 n_{g} \Delta \kappa_{g}+2 \kappa_{g} \Delta n_{g},
\end{aligned}
$$

where we assumed that $\Delta n_{g}$ and $\Delta \kappa_{g}$ are small compared to both $n_{g}$ and $\kappa_{g}$. At the frequency of the probe $(0.34 \mathrm{eV})$ the complex refractive index is given by $\tilde{n_{g}}=1.17+26.10 i$; thus, $\kappa_{g} \gg n_{g}$. It thus follows that $\Delta \varepsilon_{g, r} \propto \Delta \kappa_{g}$ and $\Delta \varepsilon_{g, i} \propto$ $\Delta n_{g}$. The change in transmittance is mostly determined by the change in the imaginary part of the refractive index $\Delta k_{g}$, whereas the change in reflectance is mostly determined by the change in the real part of the refractive index $\Delta n_{g}$. Hence, $\Delta T \propto \Delta \kappa_{g} \propto \Delta \varepsilon_{g, r}$, and $\Delta R \propto \Delta n_{g} \propto \Delta \varepsilon_{g, i}$.

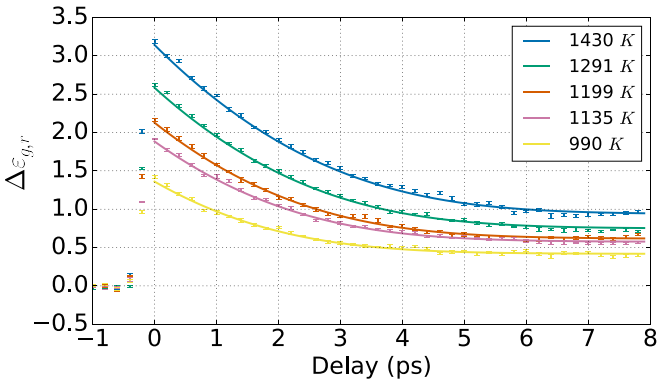

(a)

\section{Temperature dependence of the electron-electron and electron-phonon scattering rates}

Each data point in the time-dependent real and imaginary parts of the dielectric function represents a measurement of the dielectric function of gold at a particular combination of electron and lattice temperatures.

Equation (11) constitutes relatively simple relations between the electron-electron scattering rate $\gamma_{e e}$ and the electron temperature $\left[\Delta \gamma_{e e}=c\left(T_{e}^{2}-300 \mathrm{~K}^{2}\right)\right]$ and between the electron-phonon scattering rate $\gamma_{e p}$ and the lattice temperature $\left[\Delta \gamma_{e p}=b\left(T_{l}-300 \mathrm{~K}\right)\right]$. We can test the validity of the expression for $\gamma_{e e}$ using the data points of the different curves in Fig. 5. For each data point, we use the values of the electron temperature, the lattice temperature, $\gamma_{e p}$, and $\varepsilon_{g, \infty}$ resulting from the overall modeling, and we calculate the value of $\gamma_{e e}$ that is required to obtain the experimental real and imaginary dielectric functions of that data point. By repeating this procedure for all data points of all measured curves (different excitation energies), we obtain $\gamma_{e e}$ values over a large range of electron temperatures. In Fig. 6(a) we plot $\gamma_{e e}$ as a function of the electron temperature together with the theoretical expression of Eq. (11). It is seen that the obtained values of $\gamma_{e e}$ follow quite closely the quadratic dependence on the electron temperature that is given by Eq. (11), thus validating this expression.

In a similar manner we can determine the electron-phonon scattering rate $\gamma_{e p}$ from each data point of Fig. 5, using the values of the electron temperature, the lattice temperature, $\gamma_{e e}$, and $\varepsilon_{g, \infty}$ resulting from the overall modeling. In Fig. 6(b) the resulting $\gamma_{e p}$ is presented as a function of the lattice tempera-

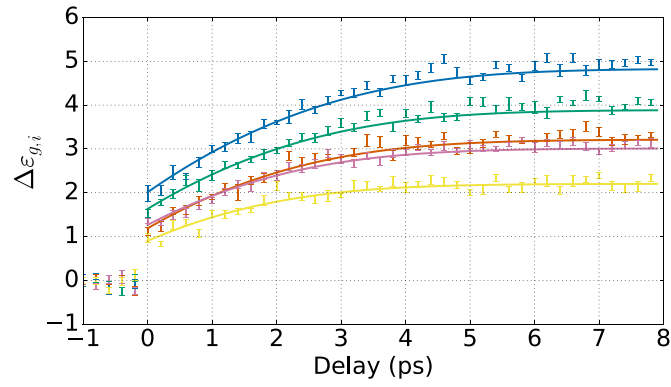

(b)

FIG. 5. The time-dependent change in (a) the real part and (b) the imaginary part of the dielectric function at $0.34 \mathrm{eV}$ calculated from the data shown in Fig. 2 for different excitation energies. The legend shows the corresponding electron temperature directly after excitation. 


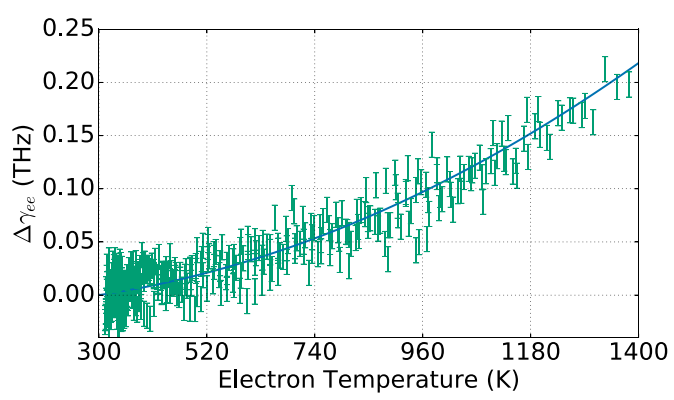

(a)

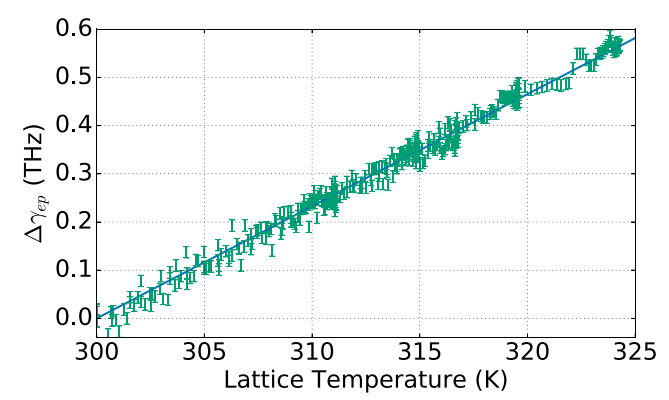

(b)

FIG. 6. (a) The dependence of $\gamma_{e e}$ on the electron temperature and (b) the dependence of $\gamma_{e p}$ on the lattice temperature. The points are obtained using the procedure described in the text, and the solid lines represent the relations between $\gamma_{e e}$ and $T_{e}$ and between $\gamma_{e e}$ and $T_{l}$ of Eq. (11).

ture and compared with the theoretical expression of Eq. (11). The thus obtained values of $\gamma_{e p}$ follow quite closely the linear dependence on the lattice temperature of Eq. (11).

\section{DISCUSSION}

We observed that the excitation of a gold film with an intense laser pulse leads to strong transient changes in the electron and lattice temperature, which in turn change the dielectric function of the film. As the dielectric function determines the reflectance and transmittance of the film, the excitation pulse will thus modify the transmittance and reflectance and thereby the absorptance. The absorptance is given by $A=1-R-T$ and can be approximated by $A\left(T_{e}, T_{l}\right) \approx A_{0}\left[1+2.69 \times 10^{-3} \times\left(T_{l}-300 \mathrm{~K}\right)+1.80 \times\right.$ $\left.10^{-8} \times\left(T_{e}^{2}-300 \mathrm{~K}^{2}\right)\right]$, where $A_{0}=0.007$ and is the absorptance at $300 \mathrm{~K}$. Thus, the absorptance increases as a function of the electron and lattice temperatures. For light incident on gold, the change in the absorptance $A$ primarily results from the change in the reflectance $R$, which decreases with increasing electron and lattice temperatures. The extinction coefficient $\kappa$ of the gold film also decreases with increasing electron and lattice temperatures, leading to an increased transmittance $T$ as a function of the electron and lattice temperatures. However, $T$ is already very small compared to $R$, and therefore, the change in $A$ will be mainly determined by the change in $R$. As the optical properties of gold for photons with an energy less than the interband transition threshold at $2.4 \mathrm{eV}$ and greater than the electron relaxation rate of $0.03 \mathrm{eV}$ depend only weakly on the photon energy, the increase of $A$ as a function of the electron and lattice temperatures occurs for all photons which satisfy these energy criteria.

When a laser pulse excites a gold film, the electron and lattice temperatures, and thus also the absorptance, increase during excitation, as excitation takes place in a finite time window, i.e., $\sim 100$ fs. From this, we expect that a selfinduced increase of the absorptance during excitation takes place. To determine the magnitude of the self-enhancement of the absorptance, we solve again the TTM, but instead of taking an instantaneous temperature difference between the electron and lattice, we include a source term $S(t)=$ $A\left(T_{e}(t), T_{l}(t)\right) P(U, t)$ in Eq. (12), where $A\left(T_{e}, T_{l}\right)$ is the absorptance that depends on the instantaneous electron and lattice temperature and $P(U, t)$ is the intensity of the excita- tion pulse (Gaussian envelope of $100 \mathrm{fs}$ FWHM) at the sample at time $t$, with a total pulse energy of $U$. The time-integrated fraction of absorbed energy of the excitation pulse is equal to $\frac{\Delta U_{e}+\Delta U_{l}}{U}$, where $\Delta U_{e}$ and $\Delta U_{l}$ are the changes in the electron and lattice energies after the excitation. We find that in order to reach an electron temperature of $5000 \mathrm{~K}$, we need a fluence of $1600 \mathrm{Jm}^{-2}$ when we take the self-induced increase of the absorptance into account, whereas a fluence of $2500 \mathrm{~J} \mathrm{~m}^{-2}$ is needed when no self-induced increase of the absorptance is taken into account. This corresponds to an increase of $\sim 55 \%$ in the energy that is absorbed.

In the analysis of the experiments presented here we did not take into account the self-induced enhancement of the absorptance and we calculated the initial rise in electron temperature using the unperturbed dielectric function. This approach is valid since at the highest used fluence in our experiments, $170 \mathrm{~J} \mathrm{~m}^{-2}$, the maximum self-induced change in the absorptance is $\sim 4 \%$. This change is within the uncertainty of the calculated absorbed energy using the unperturbed dielectric function.

The electron-phonon coupling of $\mathrm{Au}$ has been studied before, and a wide range of values for the electron-phonon coupling constant have been reported, ranging from $1.4 \times$ $10^{16}$ to $4.0 \times 10^{16} \mathrm{~W} \mathrm{~m}^{-3} \mathrm{~K}^{-1}[13]$. This wide range of reported values can be explained from the different sample preparation methods that have been employed, as the method of sample preparation determines the film thickness and the grain size within the film. These properties in turn affect the relaxation dynamics of the electrons. There have been several studies of the effect of interface and grain-boundary scattering on $G$, but these experiments yielded contradictory results $[13,28,29]$. Here we find $G=1.97 \pm 0.15 \times$ $10^{16} \mathrm{~W} \mathrm{~m}^{-3} \mathrm{~K}^{-1}$, which is thus well within the range of reported values of $G$.

The electron-phonon scattering rate $\gamma_{e p}$ depends linearly on the lattice temperature: $\gamma_{e p}=b\left(T_{l}-300 \mathrm{~K}\right)$, with $b=$ $\gamma_{0}\left(3.18 \times 10^{-3} \pm 2 \% \mathrm{~K}^{-1}\right)$. This temperature dependence agrees well with the results of other studies [27]. The linear dependence of the electron-phonon scattering rate on the lattice temperature is also predicted by the Bloch-Grüneisen equation [27], which relates $\gamma_{e p}$ to the phonon occupation number.

The electron-electron scattering rate depends quadratically on the electron temperature $\gamma_{e e}=c T_{e}^{2}$. For the coefficient 
$c$ we find $c=\gamma_{0}\left(1.62 \times 10^{-8} \pm 6 \% \mathrm{~K}^{-2}\right)$. The coefficient $c$ can be calculated using the Fermi-liquid theory [11], which yields $c=\gamma_{0} \times 5.74 \times 10^{-8} \mathrm{~K}^{-2}$. This theoretical value is larger than the value that we obtain from the analysis of the femtosecond data.

The above-mentioned theoretical result does not include the effect of screening of the Coulomb interaction between conduction electrons due to $d$-band electrons. This screening effect reduces the scattering rate by a factor of $\sqrt{\varepsilon_{g, \infty}}$ [30], where $\varepsilon_{g, \infty}=7.24$ is the static dielectric response due to $d$ band electrons. This screening effect decreases the theoretical result to $c=\gamma_{0} \times 2.13 \times 10^{-8} \mathrm{~K}^{-2}$, which is quite close to the value we obtain from our measurements. It should be noted that the screening due to $d$-band electrons is dependent on the electron temperature, thus making the actual temperature dependence of $\gamma_{e e}$ more complicated. However, within the investigated pump fluence and electron temperature range, the inclusion of the temperature dependence of the screening does not result in a significant deviation from a quadratic dependence of $\gamma_{e e}$ on the electron temperature.

A possible reason for the observed discrepancy between the theoretically predicted and experimentally determined temperature dependences of the electron-electron scattering rate is the presence of grain boundaries. Grain boundaries act as anisotropic scattering centers for electrons [31], and it has been shown that these scattering centers can increase the magnitude of the electron-electron scattering rate up to $50 \%$ in noble metals.

The electron-electron and electron-phonon scattering rates both depend on the shape of the Fermi surface [24]. As the Fermi surface of gold is anisotropic, the scattering rates are anisotropic as well. However, it has been shown that the anisotropy of the electron-electron scattering is very small [32] and that the electron-phonon scattering rate is only anisotropic at temperatures below the Debye temperature [33]. In our experiment, the excited electron distribution becomes thermal within $250 \mathrm{fs}$, which implies that the observed electron-electron and electron-phonon scattering rates represent orientational averages over the different directions in the gold nanocrystals that together constitute the thin gold film.

\section{CONCLUSIONS}

We performed transient transmission and reflection experiments on gold films using femtosecond mid-infrared pulse excitation and probing pulses. By modeling the transient transmission measurements with a two-temperature model, we obtained the electron-phonon coupling constant $G$ that determines the rate of electron temperature relaxation to the lattice. From the measured change in the transmittance and reflectance we determined the transient dielectric function. We modeled this transient dielectric function with a Drude model, including the electron-electron scattering rate $\gamma_{e e}$, the electron-phonon scattering rate $\gamma_{e p}$, and the nonresonant background $\varepsilon_{g, \infty}$ that results from interband transitions in the gold film. By fitting this Drude model to the transient dielectric function, we obtained an accurate determination of the temperature dependence of the electron-electron and electronlattice scattering rates.

\section{ACKNOWLEDGMENTS}

This work is part of the research program of the Netherlands Organisation for Scientific Research (NWO) and was performed at the research institute AMOLF. Part of this work has been carried out at ARCNL, a public-private partnership of UvA, VU, NWO, and ASML. The authors thank Paul Planken for useful discussions.
[1] N. Del Fatti, R. Bouffanais, F. Vallée, and C. Flytzanis, Phys. Rev. Lett. 81, 922 (1998).

[2] W. S. Fann, R. Storz, H. W. K. Tom, and J. Bokor, Phys. Rev. B 46, 13592 (1992).

[3] N. Del Fatti, C. Voisin, M. Achermann, S. Tzortzakis, D. Christofilos, and F. Vallée, Phys. Rev. B 61, 16956 (2000).

[4] R. H. M. Groeneveld, R. Sprik, and A. Lagendijk, Phys. Rev. B 51, 11433 (1995).

[5] M. Bonn, D. N. Denzler, S. Funk, M. Wolf, S.-S. Wellershoff, and J. Hohlfeld, Phys. Rev. B 61, 1101 (2000).

[6] T. Q. Qiu and C. L. Tien, Int. J. Heat Mass Transfer 35, 719 (1992).

[7] S. D. Brorson, J. G. Fujimoto, and E. P. Ippen, Phys. Rev. Lett. 59, 1962 (1987).

[8] N. Del Fatti, C. Voisin, D. Christofilos, F. Vallée, and C. Flytzanis, J. Phys. Chem. A 104, 4321 (2000).

[9] O. B. Wright, Phys. Rev. B 49, 9985 (1994).

[10] M. I. Kaganov, I. M. Lifshitz, and L. V. Tanatarov, ZhETF 31, 232 (1957) [J. Exp. Theor. Phys. 4, 173 (1957)].

[11] B. Y. Mueller and B. Rethfeld, Phys. Rev. B 87, 035139 (2013).

[12] P. B. Allen, Phys. Rev. Lett. 59, 1460 (1987).
[13] J. Hohlfeld, S.-S. Wellershoff, J. Güdde, U. Conrad, V. Jähnke, and E. Matthias, Chem. Phys. 251, 237 (2000).

[14] D. Fisher, M. Fraenkel, Z. Henis, E. Moshe, and S. Eliezer, Phys. Rev. E 65, 016409 (2001).

[15] J. Winter, S. Rapp, M. Schmidt, and H. P. Huber, Appl. Surf. Sci. 417, 2 (2017).

[16] J. K. Chen and J. E. Beraun, J. Opt. A 5, 168 (2003).

[17] S. S. Wellershoff, J. Hohlfeld, J. Güdde, and E. Matthias, Appl. Phys. A 69, 99 (1999).

[18] H. Zhang, S. A. Wolbers, D. M. Krol, J. I. Dijkhuis, and D. V. Oosten, J. Opt. Soc. Am. B 32, 1 (2015).

[19] M. Hövel, B. Gompf, and M. Dressel, Phys. Rev. B 81, 035402 (2010).

[20] W. J. Smit and H. J. Bakker, J. Chem. Phys. 139 (2013).

[21] I. H. Malitson, Appl. Opt. 2, 1103 (1963).

[22] F. Wooten, Optical Properties of Solids (Academic Press, New York, 1972), p. 260.

[23] R. L. Olmon, B. Slovick, T. W. Johnson, D. Shelton, S. H. Oh, G. D. Boreman, and M. B. Raschke, Phys. Rev. B 86, 235147 (2012). 
[24] N. W. Ashcroft and N. D. Mermin, Solid State Physics (Harcourt Inc., Orlando, USA, 1976), p. 826.

[25] R. W. Schoenlein, W. Z. Lin, J. G. Fujimoto, and G. L. Eesley, Phys. Rev. Lett. 58, 1680 (1987).

[26] Z. Lin, L. V. Zhigilei, and V. Celli, Phys. Rev. B 77, 075133 (2008).

[27] R. A. Matula, J. Phys. Chem. 8, 1147 (1979).

[28] W. Ma, H. Wang, X. Zhang, and W. Wang, Int. J. Thermophys. 34, 2400 (2013).
[29] J. L. Hostetler, A. N. Smith, D. M. Czajkowsky, and P. M. Norris, Appl. Opt. 38, 3614 (1999).

[30] C. Voisin, D. Christofilos, P. A. Loukakos, N. Del Fatti, F. Vallée, J. Lermé, M. Gaudry, E. Cottancin, M. Pellarin, and M. Broyer, Phys. Rev. B 69, 195416 (2004).

[31] M. Kaveh and N. Wiser, J. Phys. F 12, 935 (1982).

[32] V. Gasparov and R. Huguenin, Adv. Phys. 42, 393 (1993).

[33] J. M. Ziman, Phys. Rev. 121, 1320 (1961). 